\title{
Tradução e validação do SAQ [Sports Attitudes Questionnaire) para jovens praticantes desportivos portugueses com idades entre os 13 e os 16 anos
}

\author{
Carlos E. Gonçalves ${ }^{1}$ \\ Manuel J. Coelho e Silva ${ }^{1}$ \\ Nikos Chatzisarantis ${ }^{2}$ \\ Martin J Lee ${ }^{3}$ \\ Jaume Cruz ${ }^{4}$
}

https://doi.org/10.5628/rpcd.06.01.38

\author{
${ }^{1}$ Universidade de Coimbra \\ Faculdade de Ciências do Desporto e Educação Física \\ Portugal \\ 2 University of Exeter \\ United Kingdom \\ ${ }^{3}$ University of Exeter \\ School of Education \\ United Kingdom \\ ${ }^{4}$ Universitat Autònoma de Barcelona \\ Facultad de Psicologia \\ Espanha
}

\section{ABSTRACT}

Translation and Validation of the Sports Attitudes Questionnaire (SAQ) Applied to Young Portuguese Athletes Aged 13 to 16 Years de proceder à tradução do Sports Attitudes Questionnaire (SAQ) o inventário foi aplicado a duas amostras independentes de atletas com idades compreendidas entre os 13 e os 16 anos. Com base nos resultados de 511 sujeitos (247 rapazes e 264 raparigas), a análise factorial exploratória identificou quatro dimensões. A análise factorial confirmatória evidenciou o ajustamento do modelo de quatro factores aos dados obtidos numa amostra de 482 sujeitos (248 masculinos e 234 femininos). Em resumo, a versão portuguesa do questionário de atitudes face ao desporto parece apta a ser utilizada em futuras pesquisas.

Palavras-chave: atitudes, validade, análise factorial confirmatória.
This study examines the assessment of the educational role of youth organized sports. After obtaining the Portuguese version of the Sports Attitudes Questionnaire (SAQ), the inventory was applied on two samples of 13- to 16-years-old athletes. Based on 511 subjects (247 boys, 264 girls) exploratory factor analysis identified four dimensions. In addition, confirmatory factory analysis showed the consistency between the 4-factor model and the data collected from 482 subjects (248 males, 234 females). In summary, the Portuguese version of SAQ suggested to be a reasonable instrument for future research.

Key Words: attitudes, validity, confirmatory factor analysis. 


\section{INTRODUÇÃO}

A prática desportiva das crianças e jovens é fomentada e incentivada devido às suas virtudes formativas carácter, disciplina, vontade, de "preparação para a vida" e pró-sociais. Assistimos nas últimas décadas a um vertiginoso aumento da oferta desportiva para idades cada vez mais baixas, chegando-se à actual situação em que a participação em actividades desportivas representa a maior fatia de ocupação de tempo livre, não lectivo, entre as crianças e os adolescentes [6, 27].

No âmbito do que chamamos "desporto organizado", com praticantes regularmente inscritos em federação desportiva ou escolar e estando, ou não, orientado para o rendimento, é aceite que é a correcta organização e orientação das actividades que determina o efeito pedagógico positivo da participação das crianças e jovens. Todavia, outros estudos sugerem que o impacto da prática desportiva nos jovens pode não ser gerador de convicções e atitudes pró-sociais $[8,16]$.

Medir e avaliar as atitudes dos jovens face ao desporto tem sido uma preocupação dos investigadores, em especial a partir da última metade da década de 90 do século XX [28, 34]. O problema reside não só na dificuldade de encontrar instrumentos adequados ao objecto de estudo, como em estabelecer um enquadramento conceptual que torne evidente a expressão de condutas que os jovens praticantes julgam como as mais adequadas às situações desportivas e permitam, ao mesmo tempo, a intervenção dos agentes de ensino.

As teorias sócio-cognitivas e estruturalista têm procurado criar um quadro teórico que viabilize a investigação do modo como o raciocínio moral se forma e se transforma em condutas observáveis [34]. As investigações produzidas permitiram entrever a complexidade dos processos cognitivos e sociais que, nos diversos contextos, vão determinar as decisões morais de crianças e jovens.

No campo específico do desporto, entende-se que o comportamento dos atletas se deveria pautar pelo respeito pelo Desportivismo (sportspersonship ou fair play), conceito que englobaria um conjunto de "boas práticas", que confeririam um carácter educativo ao treino e à competição. Cruz et al. [7] afirmam que o
Desportivismo se compõe de: respeito pelas regras e pelos adversários; igualdade de condições entre os intervenientes; renúncia à vitória a todo o custo; dignidade nas vitórias e nas derrotas; "dar o melhor de si mesmo".

Para responder à questão de avaliação do construto Desportivismo, Vallerand et al. [33] propõem a Multidimensional Sportspersonship Orientations Scale MSOS-25. O instrumento baseia-se nas cinco dimensões do Desportivismo, propostas por Vallerand et al. [32]: empenhamento na participação; respeito pelas convenções sociais; respeito pelas regras e pelos árbitros; respeito pelo adversário; abordagem negativa do desportivismo. Cada dimensão é representada por cinco itens.

Na mesma linha, mas partindo da sua própria definição de fairplay, Boixadós \& Cruz [1] desenvolvem um instrumento de medida, a Escala d'Actituds de Fair Play (EAF/C), composta por 22 itens e destinada a medir as atitudes dos atletas face à sua prática desportiva. Num estudo com futebolistas federados, com idades entre os 13 e os 19 anos, foram identificados três factores: ganhar; jogo duro; espírito do jogo $e$ divertimento.

Por seu lado, e respondendo a uma solicitação do Conselho da Europa e do Sports Council do Reino Unido, que visava o estudo da ética na prática desportiva infanto-juvenil, Lee [17] coordenou uma pesquisa que deveria conduzir à elaboração de questionários de valores e atitudes, direccionados para o desporto de jovens.

O modelo adoptado $[17,19,35]$ para estudar e avaliar as premissas morais individuais que determinam as decisões, parte do princípio que as atitudes são contingentes à situação dada e informam sobre o comportamento que o atleta assumiria face a um potencial conflito moral [23]. Neste sentido, as atitudes representariam, para o atleta, a avaliação afectiva e instrumental da conduta a adoptar $[25,4]$. Partindo da identificação de atitudes efectuada por um grupo de peritos, Lee [17] elaborou o Sports Attitudes Questionnaire (SAQ), de 26 itens, que foi aplicado a uma amostra de 1391 atletas, dos 12 aos 16 anos, rapazes e raparigas, praticantes dos desportos mais populares no Reino Unido. O questionário compreende quatro escalas: Batota, Anti-desportivismo, 
Vitória a todo o preço e $A$ vitória não é o mais importante. Em estudo posterior, Lee $e t$ al. [19] modificam o SAQ de modo a que duas sub-escalas do MSOS-25 proposto por Vallerand et al. [33] - empenhamento na participação, respeito pelas convençôes sociais - substituam as escalas $A$ vitória não é o mais importante $\mathrm{e}$ Vitória a todo o preço da versão original. Os itens do questionário são reduzidos para 23 , resultando numa proposta de modelo de quatro factores, sendo dois considerados socialmente positivos (Empenhamento e Convenção) e dois socialmente negativos (Batota e Anti-desportivismo). O instrumento é aplicado a uma amostra de 549 atletas, dos 13 aos 16 anos, rapazes e raparigas, praticantes dos desportos mais populares no Reino Unido [19, 35]. Neste estudo, a consistência interna do modelo de quatro factores revela-se elevada; o coeficiente do alfa-decronbach para o Empenho foi .68, para a Convenção .82, para a Batota .81 e para o Anti-desportivismo .84 .

O Sports Attitudes Questionnaire foi aplicado a amostras de idades e de composição de demografia desportiva similares à população-alvo do presente estudo (dos 13 aos 16 anos, que, para além de atravessar o período da adolescência, corresponde igualmente à etapa de especialização na maioria das modalidades desportivas) e a sua validade psicométrica foi verificada no Reino Unido [19]. Do mesmo modo, o modelo de quatro dimensões, sendo duas consideradas socialmente positivas e duas consideradas socialmente negativas, para além de demonstrar sólida consistência interna, está em consonância conceptual com os instrumentos propostos por Vallerand [33] ou Boixadós \& Cruz [1].

O presente estudo pretende produzir a versão portuguesa do instrumento do Sports Attitudes

Questionnaire (SAQp), validando a sua estrutura multidimensional para atletas portugueses com idades compreendidas entre os 13 e os 16 anos.

\section{METODOLOGIA}

Tradução do questionário Sports Attitudes Questionnaire Com o objectivo de tornar o instrumento de pesquisa a utilizar linguística e conceptualmente acessível à população-alvo, foram seguidos um certo número de procedimentos: a) Constituição de um painel de cinco peritos com formação avançada em ciências do desporto, leitores habituais de bibliografia científica em língua inglesa, com experiência de missões de estudo e ensino no estrangeiro e/ou frequência de congressos científicos em língua inglesa com apresentação de comunicações. b) Constituição de um painel de cinco licenciados em línguas e literatura inglesa ou títulos correlatos e com especialização em técnicas de tradução.

c) Análise do trabalho efectuado pelos dois painéis de peritos, escolhendo o item candidato à escala final. Esta tarefa foi efectuada por um doutorado em Ciências do Desporto, bolseiro por um semestre em universidade dos Estados Unidos da América e por um elemento do Instituto do Desporto de Portugal, licenciado em Educação Física, com vasta experiência na tradução de artigos para a revista Treino Desportivo.

As várias fases de confrontação de alternativas de tradução, que filtram todos os itens e a experiência dos peritos, tanto no domínio da língua inglesa, como nas ciências do desporto, nomeadamente no treino de jovens, garantiram uma correcta adequação do questionário à capacidade de compreensão dos destinatários. Os curricula dos tradutores e dos especialistas em ciências do desporto são apresentados na Tabela 1.

O Sports Attitudes Questionnaire (SAQ) [17], de 23 itens, é introduzido por um breve texto: "Here are some things that some young athletes have said about the way they play sport. Please read each one and circle one of the numbers beside it to show how much you agree or disagree with it". A escala de respostas varia entre 1 e 5 : 1, I strongly disagree with the statement, 2, I disagree with the statement but not strongly, 3, I neither agree nor disagree with the statement, 4, I agree with the statement but not strongly, 5, I strongly agree with the statement.

Os itens do questionário são: (1) I go to every practice, (2) Sometimes I waste time to unsettle the opposition, (3) I would cheat if I thought it would help me win, (4) I congratulate the opposition after I have lost, (5) If other people are cheating, I think I can do, (6) I always try my best, (7) It is not against the rules to "psyche" people out so it is OK to do, (8) I shake hands with the opposition's coach, (9) I cheat if I can get away with it, (10) I sometimes try 
Tabela 1: Notas curriculares dos elementos que participaram na tradução dos questionários.

\begin{tabular}{|c|c|c|}
\hline & & Nota curriculares \\
\hline Tradutor & $\begin{array}{l}4 \\
5\end{array}$ & $\begin{array}{l}\text { Licenciatura em Filosofia Germânica pela Faculdade de Letras da Universidade do Porto. } \\
\text { Licenciatura em Línguas Estrangeiras e Literaturas Modernas Germânicas, variante de Inglês-Alemão, } \\
\text { pela Faculdade de Letras da Universidade de Coimbra. } \\
\text { Licenciatura em Línguas e Literaturas Modernas, variante de Estudos Franceses e Ingleses (via } \\
\text { científica), pela Faculdade de Letras da Universidade de Coimbra. } \\
\text { Certificado em Língua Inglesa pela University of Cambridge. } \\
\text { Licenciatura em Línguas e Literaturas Modernas, variante de Estudos Ingleses e Alemães, pela } \\
\text { Faculdade de Letras da Universidade de Coimbra. } \\
\text { Licenciatura em Línguas e Literaturas Modernas, variante de Estudos Ingleses e Alemães, pela } \\
\text { Faculdade de Letras, da Universidade de Coimbra. }\end{array}$ \\
\hline Especialista & $\begin{array}{l}2 \\
3 \\
4\end{array}$ & $\begin{array}{l}\text { Mestre em Psicologia do Desporto e participante em eventos profissionais e científicos, realizados no } \\
\text { estrangeiro, com apresentação de comunicações em Língua Inglesa. } \\
\text { Doutoramento em Ciências do Desporto, obtido numa Universidade no Reino Unido. } \\
\text { Mestre em Biocinética e Desenvolvimento, bolseiro por um semestre numa Universidade nos Estados } \\
\text { Unidos da América. } \\
\text { Mestre em Treino Desportivo de Alto Rendimento, com apresentação de comunicações em } \\
\text { congressos internacionais em língua inglesa, titular de publicações em língua inglesa, docente } \\
\text { universitário e doutorando em Ciências do Desporto. } \\
\text { Licenciado em Educação Física e Desporto e participante em eventos profissionais e científicos } \\
\text { realizados no estrangeiro, com apresentação de comunicações em língua inglesa. }\end{array}$ \\
\hline Peritos & $\begin{array}{l}1 \\
2\end{array}$ & $\begin{array}{l}\text { Doutorado em Ciências do Desporto, bolseiro por um semestre numa Universidade nos Estados Unidos } \\
\text { da América. } \\
\text { Licenciado em Ciências do Desporto, tradutor experiente em artigos da principal revista portuguesa de } \\
\text { treino desportivo. }\end{array}$ \\
\hline
\end{tabular}

to "wind up" the opposition, (11) I am always thinking about how to improve, (12) I congratulate the opposition for a good play or performance, (13) Sometimes I have to cheat, (14) I think I can unsettle my opponents as long as I don't break the rules, (15) I make an effort even if I am certain of losing, (16) It is OK to cheat if nobody knows, (17) I shake hands with the opposition-win or lose, (18) If I don't want another person to do well, then I put them off a bit, (19) Sometimes I cheat to gain an advantage, (20) It's a good idea to upset your opponents, (21) I don't give up after mistakes, (22) I congratulate the opposition after I have won, (23) I try to get officials to rule in my favour even when they shouldn't.
A Tabela 2 apresenta a solução final de itens em língua portuguesa, apurada pelo painel final de peritos. Oito dos vinte e três itens da versão final do questionário foram propostos pelo painel final de peritos. Apenas um item é exclusivamente proveniente da proposta feita pelo painel de tradutores e onze outros itens resultam da tradução efectuada pelos especialistas em ciências do desporto. Existem três afirmações que são simultaneamente sugeridas pelos especialistas e tradutores. 
Tabela 2: Proposta final da versão portuguesa do questionário de atitudes face ao desporto (SAQp].

\begin{tabular}{|c|c|c|c|c|}
\hline & & $P$ & $T$ & $\mathrm{PF}$ \\
\hline 1. & Vou a todos os treinos & 1.5 & $1 \cdot 6$ & \\
\hline 2. & Às vezes perco tempo a perturbar os adversários & 4 & & \\
\hline 3. & Era capaz de fazer batota se isso me ajudasse a ganhar & 6 & & \\
\hline 4. & Cumprimento os adversários após uma derrota & & & $\mathrm{x}$ \\
\hline 5. & Se os outros fazem batota, penso que também o posso fazer & & & $\mathrm{X}$ \\
\hline 6. & Dou sempre o meu melhor & $1,3,5$ & 3.4 & \\
\hline ?. & Como não é contra as regras pressionar psicologicamente os adversários, posso fazê-lo & & & $\mathrm{x}$ \\
\hline 8. & Cumprimento o treinador adversário & $1 \cdot 2,4$ & $3,5-6$ & \\
\hline 9. & Faço batota se ninguém der por isso & 5 & & \\
\hline 10. & Porvezes tento enganar os meus adversários & 5 & & \\
\hline 11. & Estou sempre a pensar em como melhorar & & & $x$ \\
\hline 12. & Felicito os adversários por um bom jogo ou por um bom desempenho & & 1 & \\
\hline 13. & Porvezes é preciso fazer batota & 3 & & \\
\hline 14. & Penso que posso perturbar os adversários desde que não viole as regras & $2 \cdot 3$ & & \\
\hline 15. & Esforço-me sempre, mesmo que saiba que vou perder & 3 & & \\
\hline 16. & Não há problema em fazer batota se ninguém notar & & & $x$ \\
\hline 17 . & Seja qual for o resultado, cumprimento os meus adversários & 5 & & \\
\hline 18. & Se não quiser que alguém jogue bem, tento perturbá-lo um pouco & & & $x$ \\
\hline 19. & Por vezes faço batota para obter vantagem & $3 \cdot 5$ & & \\
\hline 20. & É uma boa ideia irritar os meus adversários & & & $x$ \\
\hline 21. & Não desisto, mesmo depois de ter cometido erros & 3 & & \\
\hline 22. & Depois de ganhar, cumprimento os meus adversários & 5 & & \\
\hline 23. & Tento que os árbitros decidam a meu favor, mesmo que não seja verdade & & & $x$ \\
\hline
\end{tabular}

$P$ (peritos), $T$ (tradutores), PF (painel final).

\section{Amostra}

Lee et al. [19] conduziram tanto a análise factorial exploratória (AFE), como a AFC nos seus estudos. No entanto, dado que se trata de um estudo transcultural (uma vez que o SAQp ainda não fora aplicado a amostras portuguesas), foi decidido usar a AFE e a AFC com base em amostras independentes.

Trata-se de uma estratégia comum para a especificação de modelos, em que a AFE demonstra a sua utilidade, quando conjugada com a AFC [36].

Para a análise factorial exploratória foi efectuada uma primeira colecção de dados sobre uma amostra de 511 sujeitos dos 13 aos 16 anos (15.0 1.0 anos),
247 rapazes e 264 raparigas, 271 praticantes de desporto escolar e 240 atletas federados, 275 praticantes de modalidades individuais (atletismo, badminton, ginástica, judo, natação, ténis, ténis de mesa) e 236 de modalidades colectivas (andebol, basquetebol, futebol, voleibol).

Para a análise factorial confirmatória foram inquiridos 482 praticantes com idades compreendidas entre

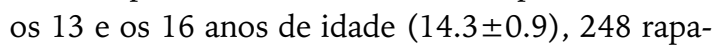
zes, 234 raparigas, sendo 167 escolares e 315 praticantes de desporto federado, num total de 222 atletas de modalidades individuais (atletismo, badmin- 
ton, canoagem, judo, ginástica, natação, remo, ténis de mesa) e 260 de modalidades colectivas (andebol, basquetebol, futebol e voleibol). Note-se que esta amostra é independente da utilizada na análise factorial exploratória.

A obtenção das amostras seguiu o princípio de combinar em proporções semelhantes atletas de desporto federado e de desporto escolar, de modalidades individuais e de modalidades de equipa, de rapazes e de raparigas, tendo-se optado sempre pela inclusão de mais do que um núcleo/clube por cada grupo de modalidade/sexo.

A aplicação dos questionários aos alunos das escolas foi precedida pela celebração de um protocolo com a Direcção Regional de Educação do Centro. Os procedimentos de recolha de dados foram registados na Comissão Nacional de Protecção de Dados, que autorizou a sua aplicação depois de verificar as condições de recolha e manuseamento. Os questionários foram preenchidos antes ou após treinos ou competições, na presença de um investigador.

\section{Tratamento estatístico}

A análise factorial pode ser conduzida no modo exploratório ou no modo confirmatório. No primeiro tipo de análise, procura-se descrever e sintetizar os dados agrupando as variáveis que se encontram correlacionadas entre si. As variáveis podem ou não ter sido seleccionadas na expectativa de encontrar uma estrutura latente já conhecida. A técnica procura responder às seguintes questões: (a) Quantos factores estão incluídos num conjunto inicial de variáveis? (b) Que interpretação para os factores? (c) Que porções da variância são explicadas por cada uma das dimensões? A extracção de factores foi efectuada após rotação varimax. Considerámos o valor crítico de $\geq 0.40$ de carga factorial para incluir uma variável num determinado factor [21]. Para a AFE foi utilizado o programa SPSS, versão12.0.

A análise factorial confirmatória limita-se a rejeitar ou aceitar um modelo de agregação das variáveis aprioristicamente estabelecido. A técnica testa o ajustamento entre os dados e o modelo teórico [10, $22,24,29]$. A existência de pesquisas anteriores per- mitiu aplicar esta técnica. De facto, Lee et al. [19] conduziram tanto a AFE, como a AFC nos seus estudos. Na AFC, foi usado o método de estimativa maximum likelihood (ML).

É desejável que o Satorra-Bentler Scaled qui-quadrado seja não significativo [14, 25]. Contudo, a estatística do qui-quadrado mostra-se sensível ao efeito da dimensão amostral, pelo que não dispensa a observação de outros indicadores complementares da bondade de ajustamento. Nessa medida, alguns autores [25], depois de terem encontrado um quiquadrado significativo, recorrem aos índices RCFI, NNFI, RMSEA e SRMR. Noutro estudo [9], depois de obterem um qui-quadrado significativo, optaram pelos índices GFI, NNFI, CFI e RMSEA. A propósito da escolha dos índices, Tabachnik \& Fidell [30] escrevem "the issue of which indices to report is a matter of personal preference, and perhaps, the preference of the journal editor", acrescentando ainda "often multiple indices are reported, and if the results of the fit indices are inconsistent, the model should probably be re-examined". No presente estudo, apoiar-nos-emos nas estatísticas providenciadas pelo pacote EQS, versão 5.7, a saber: RCFI (com um valor de corte $>.95$ ), NNFI (com um valor de corte $>.95$ ), RMSEA (com um valor de corte <.06), SRMR (com um valor de corte <.08), GFI (com um valor de corte $>.90$ ) e CFI (com um valor de corte $>.95$ ).

\section{RESULTADOS}

\section{Análise factorial exploratória}

A extracção de factores foi precedida pelo teste de Bartlett, que mostrou a adequação da técnica à solução inicial de 23 itens $\left[\chi^{2}{ }_{(253)}=5386.020, \mathrm{p} \leq .01\right]$. A análise foi realizada com recurso à rotação varimax $\mathrm{e}$ um nível de inclusão de factor loadings $\geq 0.40$, valor de corte recomendado por Pedhazur [21], mostrando uma estrutura de quatro dimensões, sem itens ambíguos. A solução final de quatro factores explica $58 \%$ da variância. É ainda de notar que todos os factores compreendem mais do que dois itens. 
Tabela 3: Análise de componentes principais do "Sport Attitudes Questionaire" (SAQ). Comunalidades e cargas factoriais dos itens após rotação varimax $(n=511]$.

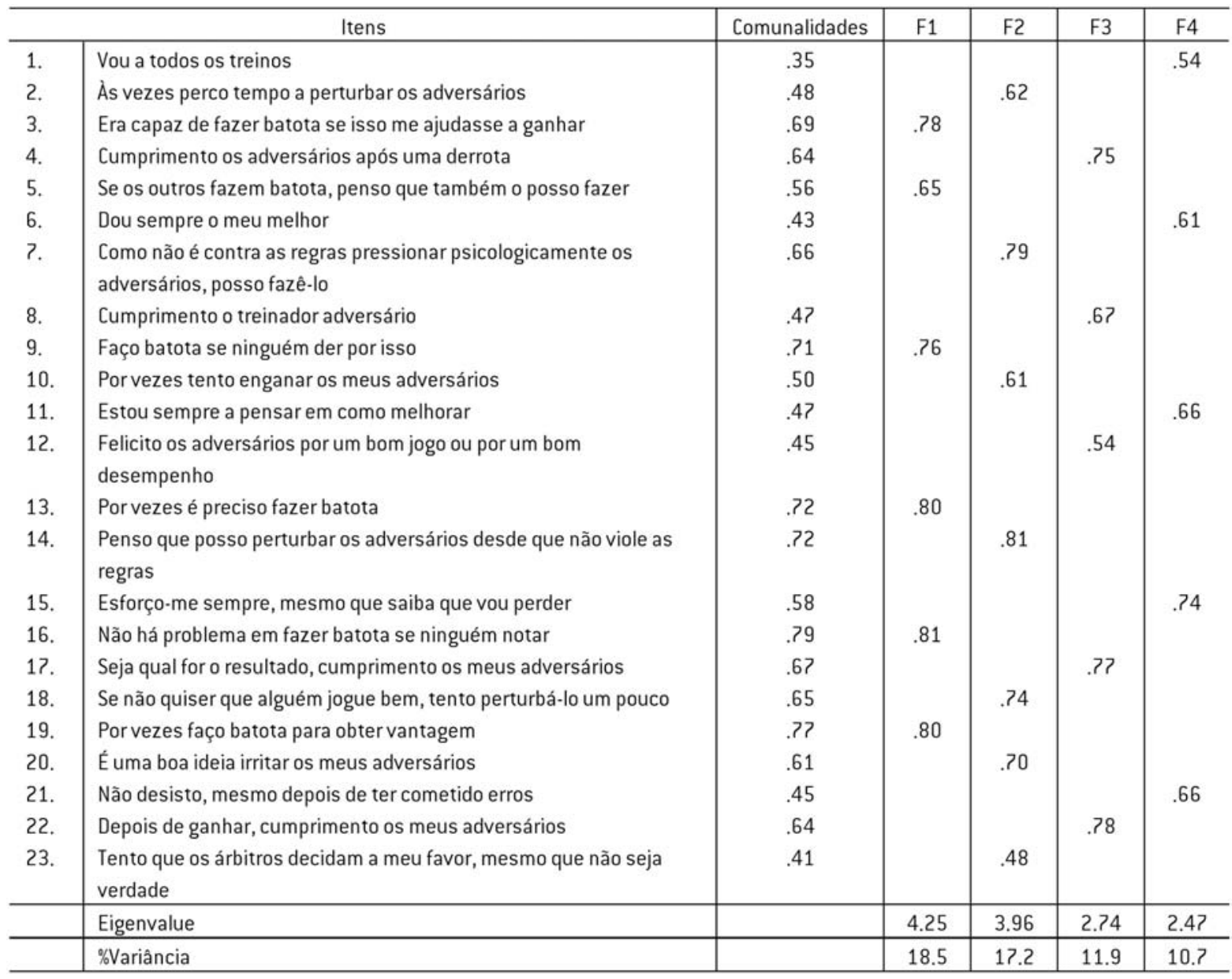

O primeiro factor (F1) pode ser interpretado como Batota e explica $19 \%$ da variância. Nele têm carga superior a 0.40 os itens 16 ("não há problema em fazer batota se ninguém notar"), 19 ("por vezes faço batota para obter vantagem"), 13 ("por vezes é preciso fazer batota"), 3 ("era capaz de fazer batota se isso me ajudasse a vencer"), 9 ("faço batota se ninguém der por isso"), 5 ("se os outros fazem batota, penso que também o posso fazer").

O segundo factor (F2) pode ser designado Anti-desportivismo e resulta da agregação do item 14 ("penso que posso perturbar os adversários desde que não viole as regras"), item 10 ("por vezes tento enganar os adversários"), item 7 ("como não é contra as regras pressionar psicologicamente os adversários, posso fazê-lo"), item 18 ("se não quiser que alguém jogue bem, tento perturbá-lo um pouco"), item 20 ("é uma boa ideia irritar os meus adversários"), item 2 ("às vezes perco tempo a perturbar os adversários"), e item 23 ("tento que os árbitros decidam a meu favor, mesmo que não seja verdade"). Esta dimensão explica $17 \%$ de variância. 
O factor (F3) designado Convenção é marcado pelos itens 22 ("depois de ganhar, cumprimento os meus adversários"), 17 ("seja qual for o resultado, cumprimento os meus adversários"), 4 ("cumprimento os adversários após uma derrota") 8 ("cumprimento o treinador adversário") e 12 ("felicito os adversários por um bom jogo ou por um bom desempenho"). Esta dimensão explica $12 \%$ da variância.
O quarto e último dos factores (F4) explica $11 \%$ da variância e parece corresponder ao domínio Empenhamento. Os itens com carga factorial acima do valor de corte estabelecido são 15 ("esforço-me sempre, mesmo que saiba que vou perder"), 11 ("estou sempre a pensar em como posso melhorar"), 21 ("não desisto mesmo depois de ter cometido erros"), 6 ("dou sempre o meu melhor") e 1 ("vou a todos os treinos").

Tabela 4: Cargas factoriais e coeficientes de consistência interna prevendo quatro itens em cada uma das componentes.

\begin{tabular}{|c|c|c|c|c|c|}
\hline \multirow{5}{*}{$\begin{array}{l}\text { Factor } \\
\text { Batota }\end{array}$} & \multicolumn{2}{|r|}{ Itens } & \multirow{5}{*}{$\begin{array}{c}\text { Carga } \\
.81 \\
.80 \\
.80 \\
.78 \\
\end{array}$} & \multirow{5}{*}{$\begin{array}{c}\text { alpha } \\
.90\end{array}$} & \multirow{5}{*}{$\begin{array}{c}\begin{array}{c}\text { alpha se } \\
\text { eliminado o } \\
\text { item }\end{array} \\
.86 \\
.86 \\
.87 \\
.89 \\
\end{array}$} \\
\hline & 16. & Não há problema em fazer batota se ninguém notar & & & \\
\hline & 19. & Por vezes faço batota para obter vantagem & & & \\
\hline & 13. & Porvezes é preciso fazer batota & & & \\
\hline & 3. & Era capaz de fazer batota se isso me ajudasse a ganhar & & & \\
\hline \multirow{4}{*}{$\begin{array}{l}\text { Anti- } \\
\text { desportivismo }\end{array}$} & 14. & $\begin{array}{l}\text { Penso que posso perturbar os adversários desde que não viole } \\
\text { as regras }\end{array}$ & .81 & \multirow{4}{*}{.85} & .80 \\
\hline & 7. & $\begin{array}{l}\text { Como não é contra as regras pressionar psicologicamente os } \\
\text { adversários, posso fazê-lo }\end{array}$ & .79 & & .81 \\
\hline & 18. & $\begin{array}{l}\text { Se não quiser que alguém jogue bem, tento perturbá-lo um } \\
\text { pouco }\end{array}$ & .74 & & .82 \\
\hline & 20. & É uma boa ideia irritar os meus adversários & .70 & & .81 \\
\hline \multirow{4}{*}{ Convenção } & 22. & Depois de ganhar, cumprimento os meus adversários & .78 & \multirow{4}{*}{.78} & .71 \\
\hline & 17. & Seja qual for o resultado, cumprimento os meus adversários & 77 & & .69 \\
\hline & 4. & Cumprimento os adversários após uma derrota & .75 & & .69 \\
\hline & 8. & Cumprimento o treinador adversário & .67 & & .80 \\
\hline \multirow{4}{*}{ Empenho } & 15. & Esforço-me sempre, mesmo que saiba que vou perder & .74 & \multirow{3}{*}{.67} & .54 \\
\hline & 21. & Não desisto, mesmo depois de ter cometido erros & .66 & & .64 \\
\hline & 11. & Estou sempre a pensar em como melhorar & .66 & & .61 \\
\hline & 6. & Dou sempre o meu melhor & .61 & & .62 \\
\hline
\end{tabular}

Como se pode ver na Tabela 4, os coeficientes de consistência interna (quadrado do coeficiente de correlação múltipla entre os itens da componente principal e os scores da componente em que os itens são incluídos) são sempre superiores a .67, valor que pode ser considerado aceitável. Atendendo aos potenciais benefícios que poderiam resultar na melhoria das propriedades psicométricas do questionário, decidimos investigar se os valores de alfade-cronbach poderiam ser aumentados a partir da eliminação de um item. De acordo com os resultados igualmente apresentados na Tabela 4, a subtracção de itens não acarretaria ganhos nos coeficientes de consistência interna.

A análise das Tabelas 4 e 5 mostra que a solução encontrada para o presente estudo pode ser considerada como bastante satisfatória, uma vez que, para cada uma das dimensões, existem sempre 2 ou 3 itens comuns aos dois estudos. Mais, não existe nenhum item que esteja numa das pesquisas agregado a uma componente principal e no outro estudo apareça como marcador de uma dimensão diferente. 
Tabela 5: Cargas factoriais após análise factorial exploratória no estudo de Lee et al. [2002]

\begin{tabular}{l|c|l|c|c}
\hline Factor & \multicolumn{2}{|c|}{ Itens } & Carga \\
\hline \multirow{4}{*}{ Cheating } & 9. & I cheat if I can get away with it & .82 \\
& 13. & Sometimes I have to cheat & .79 \\
& 16. & It is OK to cheat if nobody knows & .81 \\
\hline \multirow{5}{*}{ Gamesmanship } & 10. & I sometimes try to "wind up" the opposition & .77 \\
& 18. & If I don't want another person to do well, then I put them off a bit & .67 \\
& $?$. & It is not against the rules to "psyche" people out so it is OK & .63 \\
\hline \multirow{3}{*}{ Convention } & 12. & I congratulate the opposition for a good play or performance & .72 \\
& 22. & I congratulate the opposition after I've won & .71 \\
\hline \multirow{5}{*}{ Commitment } & 4. & I congratulate the opposition after I have lost & .66 \\
\hline & 1. & I go to every practice & .49 \\
\hline
\end{tabular}

Os resultados apresentados na Tabela 5 referem-se ao estudo desenvolvido por Lee et al. [19] com o objectivo de testar um modelo teórico da influência da estrutura de valores sobre as atitudes face à prática desportiva. A referida pesquisa foi desenvolvida numa amostra de 549 rapazes e raparigas ingleses praticantes de desporto organizado e competitivo. A análise de conteúdo dos itens que aparecem na Tabela 4 e não constam da Tabela 5, sugere que a agregação encontrada no presente estudo é adequada, ou seja: - Na primeira componente, Batota, consta o item "Por vezes faço batota para obter vantagem", não incluído na pesquisa de Lee et al. [19];

- A segunda componente, Anti-desportivismo, é composta pelos itens "É uma boa ideia irritar os adversários" e "Tento que os árbitros decidam a meu favor, mesmo que não seja verdade", que não entravam na solução de Lee et al. [19], apresentada na Tabela 5; — Os itens "Seja qual for o resultado, cumprimento os meus adversários" e "Cumprimento o treinador adversário" entram na terceira componente principal do nosso estudo, interpretada como Convenção. Estas duas expressões não tinham sido incluídas no estudo original com jovens ingleses;

- O quarto domínio, interpretado como Empenho, contém dois itens que tinham escapado à estrutura factorial proposta por Lee et al. [19]: "Estou sempre a pensar em como melhorar" e "Esforço-me sempre, mesmo que saiba que vou perder".

\section{Análise factorial confirmatória}

A partir da Tabela 4 e em concordância com as indicações dos autores da versão original do questionário de atitudes dos jovens face à prática desportiva, assumimos a existência de quatro factores (cheating, gamesmanship, convention, commitment). Foram escolhidos os itens apresentados na Tabela 6 como variáveis candidatas a serem marcadoras de cada um dos factores. Os resultados da análise factorial confirmatória relativos ao ajustamento global do modelo mostram um coeficiente de Mardia-Based Kappa $=0.31$ e um valor de Satorra Bentler Scaled $\chi^{2}{ }_{(98)}=122.2$, significativo para $\mathrm{p}<.005, \mathrm{RCFI}=.99 ; \mathrm{NNFI}=.98$; RMSEA $=.03$ (CI 90\%=.02-.04); SRMR =.04; GFI $=.96$; $\mathrm{CFI}=.98$. Estes dados mostram a adequação dos vários indicadores para considerar o modelo de quatro factores ajustado aos dados do presente estudo.

\section{DISCUSSÃO E CONCLUSÕES}

De uma forma sumária, podemos afirmar que a tradução dos 23 itens do Sports Attitudes Questionnaire (SAQ) não colocou problemas semanticamente irresolúveis, tendo sido possível obter uma solução mais económica de quatro dimensões. Os factores encontrados pela análise factorial exploratória possuem uma essência de conteúdo parcialmente semelhante à solução do estudo inicial desenvolvido no Reino Unido por Lee et al. [19]. Não foram encontrados itens problemáticos, com carga factorial em mais do que um 
Tabela 6: Parâmetros item-factor $(\rho)$ e erro de medida $[\varepsilon$ ] na estrutura de 4 dimensões do $S A \emptyset$ (Sports Attitudes Questionnaire] testada pela análise factorial confirmatória.

\begin{tabular}{l|c|c|l|c|c}
\hline Factor & $\rho$ & \multicolumn{3}{|c}{ Item } & $\varepsilon$ \\
\hline \multirow{4}{*}{ Batota } & +0.76 & 3. & Era capaz de fazer batota se isso me ajudasse a ganhar & 0.65 \\
& +0.82 & 9. & Faço batota se ninguém der por isso & 0.58 \\
& +0.73 & 13. & Por vezes é preciso fazer batota & 0.68 \\
& +0.86 & 19. & Por vezes faço batota para obter vantagem & 0.52 \\
\hline & +0.69 & 7. & Como não é contra as regras pressionar psicologicamente os adversários, posso & 0.73 \\
& & & fazê-lo & 0.65 \\
Anti- & +0.76 & 18. & Se não quiser que alguém jogue bem, tento perturbá-lo um pouco & 0.66 \\
desportivismo & +0.75 & 20. & É uma boa ideia irritar os meus adversários & 0.75 \\
\hline \multirow{4}{*}{ Convenção } & +0.67 & 23. & Tento que os árbitros decidam a meu favor, mesmo que não seja verdade & 0.82 \\
& +0.57 & 4. & Cumprimento os adversários após uma derrota & 0.85 \\
& +0.53 & 8. & Cumprimento o treinador adversário & 0.60 \\
& +0.80 & 17. & Seja qual for o resultado, cumprimento os meus adversários & 0.75 \\
\hline & +0.66 & 22. & Depois de ganhar, cumprimento os meus adversários & 0.88 \\
& +0.48 & 6. & Dou sempre o meu melhor & 0.71 \\
& +0.71 & 11. & Estou sempre a pensar em como melhorar & 0.89 \\
\hline
\end{tabular}

factor. Comparativamente ao estudo de Lee et al. [19] a presente solução oferece a vantagem de possuir coeficientes de consistência interna mais elevados e ainda de apresentar cada um dos factores marcado por 4 itens. Adicionalmente, a análise factorial confirmatória demonstra a bondade de ajustamento do modelo de quatro dimensões de quatro itens cada.

A validade do questionário para a realidade do desporto infanto-juvenil em Portugal surge confirmada através do presente estudo, viabilizando a sua aplicação, em conjunto com outros instrumentos, em investigações mais abrangentes. O estudo das atitudes, será sempre informativamente pobre, quando levado a cabo isoladamente [15].

A integração do estudo das atitudes num modelo mais compreensivo dos efeitos do treino e da competição sobre os jovens participantes, implica o recurso a outros construtos [2]. Do mesmo modo, o estudo das variáveis ecológicas (outros significantes, ambiente de clube ou escola, ambiente da equipa), com recurso a outros instrumentos e métodos, surge como uma via promissora para conhecer a realidade e intervir na prática, tal como é sugerido nos estudos de Brustad et al. [3], Guivernau \& Duda [13] e Torregosa et al. [31].

\section{AGRADECIMENTOS}

A investigação foi subsidiada pelo Programa de Apoio Financeiro à Investigação no Desporto (PAFID), do Instituto de Desporto de Portugal, $\mathrm{N}^{\circ} 223 / 2004$. A aplicação dos questionários aos alunos das escolas só foi possível através do protocolo celebrado com a Direcção Regional de Educação do Centro. Os autores agradecem a colaboração do Doutor José Carlos Leitão (UTAD) na revisão da versão inicial do presente artigo.

\section{CORRESPONDÊNCIA}

\section{Carlos Eduardo Gonçalves}

Faculdade de Ciências do Desporto

e Educação Física

Universidade de Coimbra

Estádio Universitário - Pavilhão III

3040-156 Coimbra

PORTUGAL

carlosgoncalves@fcdef.uc.pt 


\section{REFERÊNCIAS BIBLIOGRÁFICAS}

1. Boixadós M, Cruz J (1995). Construction of a fairplay attitude scale in soccer. In R. Vanfraeehem-Raway \& Y. Vanden Auweele (Eds.) Proceedings of the IX European Congress on Sport Psychology. Brussels: Belgian Federation of Sport Psychology, 4-11.

2. Boixadós M, Cruz J, Torregrosa M, Valiente L (2004). Relationships among motivational climate, satisfaction, perceived ability, and fair play attitudes in young soccer players. Journal of Applied Sport Psychology 16: 301-317.

3. Brustad R, Babkes M, Smith A (2001). Youth in sport. Psychological considerations. In R.N. Singer, H.A. Hausenblas, \& C.M. Janelle (Eds.) Handbook of Sport Psychology. New York: Wiley, 604-635.

4. Chantal Y, Robin P, Vernat J-P, Bernache-Assolant I (2005). Motivation, sportspersonship, and athletic aggression: a mediational analysis. Psychology of Sport and Exercise 6: 233-249.

5. Coelho e Silva M, Ribeiro L, Barros F, Figueiredo A, Gonçalves CE (2004). Abandono da Prática de Basquetebol Competitivo e Organizado. Manuscrito submetido para publicação.

6. Cruz J, Boixadós M, Torregrosa M, Valiente L, Villamarin F (2001). Values, socio-moral attitudes and achievement goals among youth team sports participants in Spain. In A. Papaioannou, M. Goudas, Y. Theodorakis (Eds.) In the Dawn of the Millenium: $10^{\text {th }}$ World Congress of Sport Psychology. Skiathos: Christodoulidi Publications, 195-197.

7. Cruz J, Boixadós M, Valiente L, Torregrosa M (2001). Se pierde el "fairplay" y la deportividad en el deporte en edad escolar? Apunts: Educación Física y Deportes 64: 6-16.

8. Cruz J, Boixadós M, Valiente L, Torregrosa M, Mimbrero J (1996). Existe un deporte educativo?: papel de las competiciones deportivas en el proceso de socialización del niño. Revista de Psicologia del Deporte 9-10: 111-132.

9. Fernandes H, Vasconcelos Raposo J (2004). A teoria da auto-determinação no contexto desportivo. Horizonte 19, 114: dossier.

10. Fonseca AM (1999). Atribuições em contexto de actividade física ou desportiva - perspectivas, relações e implicações. Tese de doutoramento. Faculdade de Ciências do Desporto e de Educação Física, Universidade do Porto.

11. Gonçalves CE (2004). Desporto infanto-juvenil e educação moral -situação, constrangimentos e perspectivas. Treino Desportivo Especial 6: 68-74.

12. Gonçalves CE, Coelho e Silva M (2004). Contemporary trends and issues in youth sports in Portugal. In M Coelho e Silva \& RM Malina (Eds.) Children and Youth in Organized Sports. Coimbra: Imprensa da Universidade.

13. Guivernau M, Duda J (2002). Moral atmosphere and athletic aggressive tendencies in young soccer players. Journal of Moral Education 31 (1): 67-85.

14. Hu L, Bentler P (1999). Cutoff criteria for fit indexes in covariance structure analysis. Conventional criteria versus new alternatives. Structural Equation Modeling 6: 1-55.

15. Kavussanu M, Ntoumanis N (2003). Participation in sport and moral functioning: does ego orientation mediate their relationship? Journal of Sport \& Exercise Psychology 25: 501-518.

16. Keech M, Mcfee G (2000). Locating issues and values in sport and leisure cultures. In M. Keech, G. Mcfee (Eds.) Issues and values in sport and leisure cultures. Oxford: Meyer \& Meyer Sport, 1-24.
17. Lee MJ (1996). Young people, sport and ethics: an examination of fair play in youth sport. Technical report to the Research Unit of the Sports Council. Londres.

18. Lee M, Whitehead J, Balchin N (2000). The measurement of values in youth sport: development of the youth sport values questionnaire. Journal of Sport and Exercise Psychology 22: 307-326.

19. Lee MJ, Whitehead J, Ntoumanis N, Hatzigeorgiadis A (2002). The effect of values, achievement goals and perceived ability on moral attitudes in youth sport. Technical Report to the Economic and Social Research Council.

20. Lemyre PN, Roberts G, Ommundsen Y (2002). Achievement goal orientations, perceived ability, and sportspersonship in youth soccer. Journal of Applied Sport Psychology 14: 120-136.

21. Pedhazur EL (1982). Multiple regression in behavioural research - explanation and prediction. Fort Worth: Holt, Rinehart \& Winston.

22. Peiró C, Sanchís JM (2004). Las Propriedades Psicométricas de la Versión Inicial del Cuestionario de Orientación a la Tarea Y al Ego (TEOSQ) Adaptado a la Educación Física en su Traducción al Castellano. Revista de Psicologia del Deporte 13 (1): 26-40.

23. Prat M, Soler S (2003). Actitudes, Valores y Normas en la Educación Física y el Deporte. Barcelona: INDE.

24. Reinboth M, Duda J (2004). The motivational climate, perceived ability, and athlete's psychological and physical well-being. The Sport Psychologist 18: 237-251.

25. Reinboth M, Duda J, Ntoumanis N (2004). Dimensions of coaching behavior, need satisfaction, and the psychological and physical welfare of young athletes. Motivation and Emotion 28 (3): 297-313.

26. Rhodes R, Courneya K (2005) Threshold assessment of attitude, subjective norm, and perceived behavioral control for predicting exercise intention and behavior. Psychology of Sport and Exercise 6: 349-361.

27. Seefeldt V, Clark M (2002) The continuing evolution in youth sports: what does the future hold? In F. Smoll \& R Smith (Eds.) Children and Youth in Sport: a Biopsychosocial Perspective. Dubuque: Kendall/Hunt.

28. Shields D, Bredemeier B, Power F (2002). Character development and children's sport. In F. Smoll \& R. Smith (Eds.) Children and Youth in Sport: a Biopsychosocial Perspective. Dubuque: Kendall/Hunt, 537-563.

29. Standage M, Treasure D, Duda J, Prusak K (2003). Validity, Reliability, and invariance of the Situational Motivation Scale (SIMS) across diverse physical activity contexts. Journal of Sport \& Exercise Psychology 25: 19-43.

30. Tabachnick BG, Fidell LS (2001). Using multivariate statistics (Fourth edition). Boston: Allyn \& Bacon.

31. Torregrosa M, Figueroa J, Garcia-Mas A, Sousa C, Vilches D, Villamarin F, Cruz J (2005). La relación entre las familias (padres y madres) y el compromiso deportivo de futbolistas cadetes. Comunicação ao Congresso Sul-americano de Psicologia do Desporto. Montevideu.

32. Vallerand R, Deshaies P, Cuerrier JP, Brière N, Pelletier L (1996). Toward a Multidimensional Definition of Sportsmanship. Journal of Applied Sport Psychology 8: 89-101.

33. Vallerand R, Briere N, Blanchard C, Provencher P (1997). Development and validation of the multidimensional sportspersonship orientations scale. Journal of Sport and Exercise Psychology 16: 126-140. 
34. Weiss M, Smith A (2002). Moral development in sport and physical activity: theory, research, and intervention. In T. Horn (Ed.) Advances in Sport Psychology. Champaign, Il.: Human Kinetics, 243-280.

35. Whitehead J, Lee M, Hatzigeorgiadis (2003). Goal orientations as mediators for the personal value system. Journal of Sport Sciences 21: 4.

36. Williams J, Jerome G, Kenow L, Rogers T, Sartain T, Darland G (2003). Factor structure of the coaching behavior questionnaire and its relationship to athlete variables. The Sport Psychologist 17: 16-34. 